\title{
Clinical use of cabozantinib in the treatment of advanced kidney cancer: efficacy, safety, and patient selection
}

This article was published in the following Dove Press journal:

OncoTargets and Therapy

23 September 2016

Number of times this article has been viewed

\author{
Steven S Yu \\ David I Quinn \\ Tanya B Dorff \\ Division of Oncology, Norris \\ Comprehensive Cancer Center, \\ University of Southern California, \\ Los Angeles, CA, USA
}

Correspondence: Steven S Yu

Division of Oncology, Norris Comprehensive Cancer Center, University of Southern California, I 44 I Eastlake Avenue, Suite 3440, Los Angeles, CA 90033, USA

$\mathrm{Tel}+\mathrm{I} 3238653900$

Email steven.yu@med.usc.edu

\begin{abstract}
Clear cell (cc) renal cell carcinoma (RCC) is the most common type of cancer found in the kidney accounting for $\sim 90 \%$ of all kidney cancers. In 2012 , there were $\sim 337,000$ new cases of RCC diagnosed worldwide with an estimated 143,000 deaths, with the highest incidence and mortality in Western countries. Despite improvements in cancer control achieved with VEGF- and mTOR-targeted therapy for RCC, progression remains virtually universal and additional therapies are needed. The pivotal results of the METEOR trial led to cabozantinib's designation as a breakthrough drug by the US Food and Drug Administration and its approval for treatment of advanced RCC in 2016. Subsequent data from the CABOSUN trial, where caboxantinib is compared with sunitinib, will provide information on the relative activity of cabozantinib as first-line therapy for ccRCC. We review the development of cabozantinib in advanced RCC and its role in the treatment landscape for advanced RCC.
\end{abstract}

Keywords: cabozantinib, renal cell carcinoma, kidney cancer, clear cell carcinoma, tyrosine kinase inhibitor

\section{Introduction}

Clear cell (cc) renal cell carcinoma (RCC) is the most common type of cancer found in the kidney accounting for $\sim 90 \%$ of all kidney cancers. In 2012 , there were $\sim 337,000$ new cases of RCC diagnosed worldwide with an estimated 143,000 deaths, with the highest incidence and mortality in North America and Europe. The USA is among the top four countries in incidence and mortality worldwide. ${ }^{1,2}$ The development of RCC is associated with smoking, obesity, male sex, and family history and genetic conditions such as von Hippel-Lindau disease as well as possibly type 2 diabetes mellitus and hepatitis $\mathrm{C}$ infection. ${ }^{3,4}$ In the USA, there were an estimated 62,000 new cases and $\sim 14,000$ deaths from RCC in 2015..$^{5}$ Patients with localized disease have a nearly $92 \%$ 5-year survival with curative surgery, whereas patients with advanced RCC have 5-year survival rates of $\sim 11 \%-12 \%{ }^{6}$ Despite improvements in cancer control achieved with VEGF- and mTOR-targeted therapy for RCC, progression remains virtually universal and additional therapies are needed. In this review, we delineate the differences between cabozantinib and preexisting VEGF-targeted therapy by reviewing the efficacy, safety, and patients who benefit from cabozantinib. We assess the quality of preclinical data and Phases I-III trials testing cabozantinib in various cancer types and identify gaps in knowledge where new trials are needed. 


\section{Methods}

For the elements related to caboxantinib use in renal cell cancer for this review, we undertook a systematic assessment of literature and peer-reviewed presentations by searching PubMed, MEDLINE, and major oncology meeting (ASCO. org, ESMO.org, and ECCO.org) abstracts. The following keywords were used in the database searches: (cabozantinib or cabozantinib-s-malate or RTK inhibitor or XL 184 or tyrosine kinase inhibitor or TKI or multi-TKI) and (c-MET or MET or VEGFR or VEGFR2 or AXL or RET) and (renal cell carcinoma or kidney cancer or clear cell renal carcinoma or renal cancer) and (tumor growth or angiogenesis). Hits were confirmed as having full text or full presentation content that was accessible and vetted by the authors for relevance to the review. Content was then tabulated and summarized for use in the review. Study design, sample size, treatment effect, and adverse effects were reviewed by the three authors. The search included preclinical studies and human studies. Publications not primarily published in English were excluded.

\section{Current therapies in advanced RCC}

Prior to 2005, high-dose interleukin-2 and interferon alpha (IFN $\alpha$ ) were the only approved treatments for advanced or metastatic RCC disease with $\sim 5 \%$ of patients achieving a complete response. ${ }^{7}$ However, the use of high-dose interleukin- 2 is limited to relatively young, healthy patients and restricted to administration in centers with experience in managing the considerable toxicities of the regimen. With the recognition of the biologic basis of RCC due to loss of the von Hippel Lindau (VHL) tumor suppression of angiogenic pathways, therapeutic development focused on targeting vascular endothelial growth factor (VEGF) and mTOR pathways. The first agents to be approved were multitargeted receptor tyrosine kinase inhibitors (TKIs). Currently, the US Food and Drug Administration (FDA) approved the following five VEGF pathway inhibitors in metastatic RCC: bevacizumab, sunitinib, sorafenib, pazopanib, and axitinib. The inhibition of the VEGF/VEGFR pathway by these drugs has moved the overall survival in metastatic RCC to a median of 2 years in the first-line setting. ${ }^{8}$ In addition to targeting the VEGF receptor, these agents inhibit a variety of receptors including PDGF and c-kit.

While these TKIs significantly prolonged progressionfree survival (PFS) compared to IFN $\alpha$ in previously untreated patients with advanced or metastatic RCC, issues including toxicity led to further development of additional agents such as axitinib and pazopanib. ${ }^{9-12}$ Axitinib is highly selective for the inhibition of VEGFR-1, VEGFR-2, and VEGFR-3 and was approved by the FDA for progressive disease after one "first-line" therapy based largely on the AXIS trial. ${ }^{12}$ A Phase III randomized trial, the AXIS trial, randomized 723 patients with metastatic RCC to axitinib or sorafenib after failure of first-line therapy and demonstrated that axitinib had significantly longer PFS than sorafenib (6.7 vs 4.7 months; hazard ratio [HR] 0.665; 95\% CI 0.544-0.812; $P<0.0001)$. However, no overall survival benefit was observed. In a Phase III trial with 435 patients, pazopanib, another VEGF, PDGF, and Kit inhibitor, doubled the PFS but had no improvement in OS in locally advanced or metastatic disease when compared with placebo. ${ }^{13}$ When compared with sunitinib head-to-head in the COMPARZ trial, there was no difference in PFS or OS. ${ }^{14}$ An alternative approach to suppressing VEGF signaling was to target the ligand with bevacizumab, a monoclonal antibody that binds to circulating VEGF and prevents it from activating the VEGF receptor. This strategy was also successful with prolonged PFS in combination with interferon compared to IFN $\alpha$ alone in the AVOREN and CALGB trials, leading to FDA approval for its use in RCC..$^{15,16}$ The mTOR pathway plays a central role in the regulation of cell growth, influencing many critical molecular functions, including angiogenesis, cell proliferation, and glucose homeostasis. mTOR signaling is upregulated in RCC, making it an attractive therapeutic target. ${ }^{17}$ The following two agents were developed based on their ability to inhibit mTOR signaling: temsirolimus, an intravenous preparation administered weekly, and everolimus, an oral analog of rapamycin. A Phase III trial led to the approval of temsirolimus for previously untreated, poor-risk RCC. Six hundred twenty-six previously untreated patients were randomly assigned to temsirolimus, temsirolimus + IFN $\alpha$, or IFN $\alpha$ monotherapy. Temsirolimus significantly prolonged the median overall survival compared to IFN $\alpha$ as a single agent (10.9 vs 7.3 months, HR 0.73, 95\% CI 0.58-0.92). ${ }^{18}$ However, in VEGF-pretreated patients, temsirolimus demonstrated a shorter overall survival than sorafenib in 512 patients who had progressed on sunitinib. ${ }^{19}$ On the other hand, everolimus initially was approved based on its efficacy in VEGF-pretreated patients. In the RECORD-1 trial, a double blind, randomized, placebo-controlled Phase III trial, everolimus demonstrated PFS superiority over placebo in patients with metastatic RCC progressing on VEGF TKIs. A total of 416 patients were randomized to either everolimus or placebo, resulting in a median PFS of 4.9 months in the everolimus group vs 1.9 months in the placebo group. ${ }^{20}$ 


\section{VEGF and MET in RCC VEGF}

Angiogenesis involves the formation of new blood vessels from the preexisting blood vessels and is one of the hallmarks of cancer. Neoangiogenesis supplies the tumor with nutrients for progression and invasion into surrounding tissue and contributes to lymphatic invasion and distant metastasis. ${ }^{21}$ The process is controlled by growth factors such as vascular endothelial growth factor, fibroblast growth factor, hepatocyte growth factor, insulin-like growth factor, and transcription factors such as hypoxia inducible factor to increase endothelial cell proliferation, migration, and survival (Figure 1). ${ }^{22}$ In the majority of renal cell cancer, the VHL is inactivated causing upregulation of VEGF and also other factors. VEGF stimulates the endothelial cells, which form the walls of the vessels and help in maintaining the transport of oxygen and nutrients to the tissues, hence promoting growth. The upregulation of VEGF leads to an increased angiogenesis, endothelial permability, and tumor cell viability and a more invasive tumor phenotype..$^{23}$ Therapy with agents directed against the VEGF protein or the VEGF receptor is a central basis of current treatments with today's antiangiogenic drugs.
Within the VEGF family of glycoproteins, there are VEGF-A, VEGF-B, VEGF-C, VEGF-D, and placental growth factors, each involved in angiogenesis and formation of other vessels. ${ }^{24}$ Each glycoprotein is activated by binding to an extracellular tyrosine kinase receptor on the cell surface called VEGFR-1, VEGFR-2, and VEGFR-3. These receptors dimerize and phosphorylate, activating the cellular responses exhibited by VEGF glycoproteins..$^{25}$ VEGFR-2 is hypothesized to mediate all the cellular functions of VEGF, and VEGFR-1 seems to modulate the function of VEGFR-2, although its exact function is not defined. The most important glycoprotein in the VEGF family is VEGF-A. This ligand is particularly important because it has a dramatic upregulation of its expression levels under hypoxic conditions. During hypoxic conditions, hypoxia inducible factor is stabilized, binding to specific promoter elements, which are present in the promoter region of VEGF-A. ${ }^{26}$ This activated VEGF-A gets bound to the VEGFR, mostly with VEGFR-2, inducing angiogenesis. In preclinical models, targeting the VEGF signaling pathway has proven to be efficacious, inhibiting neovascularization and yielding tumor regression in animal models. ${ }^{27}$

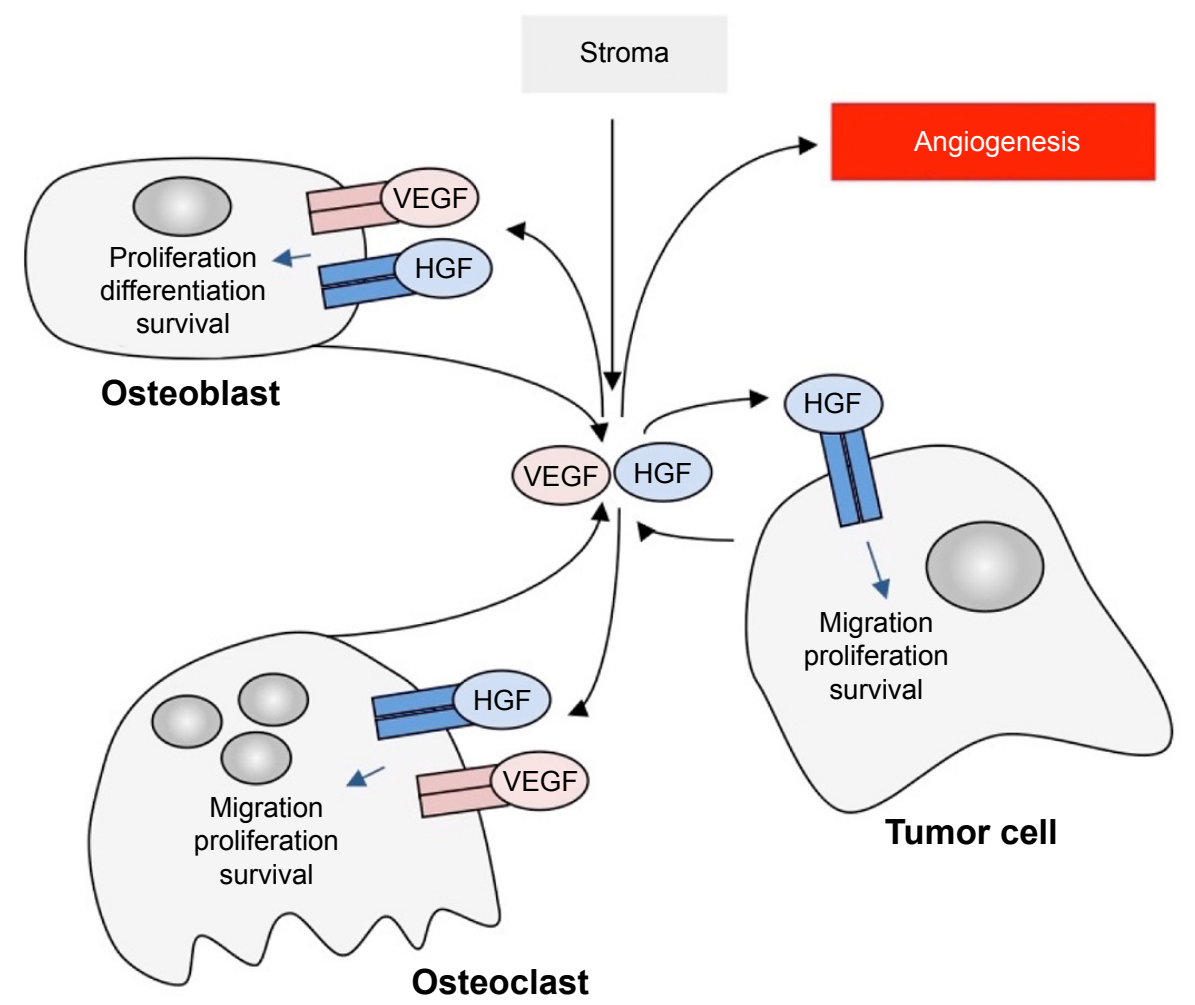

Figure I Angiogenesis is controlled by growth factors such as vascular endothelial growth factor and hepatocyte growth factor, which increase endothelial cell proliferation, migration, and survival.

Note: Adapted with permission from Exelixis, Inc. (c) $2016 .{ }^{85}$ 
Targeting the VEGF has been a very successful strategy for treating metastatic RCC. However, suppression of VEGF signaling is not universally effective; there are patients with primary refractory disease as well as nearly universal acquired resistance. Redundancy in mechanisms for angiogenesis likely account for some resistance, including induction of the protooncogene called mesenchymalepithelial transition (MET) factor. ${ }^{28,29}$ VEGF and MET are overexpressed in hypoxic environments to promote survival by stimulating angiogenesis or facilitating migration away from the hypoxic zone (Figure 2). ${ }^{30-32}$ Inhibition of the VEGF pathway alone can induce hypoxemia and also trigger a compensatory upregulation of MET expression, which helps drive tumor invasion. ${ }^{25}$ Targeting the MET is the rational strategy to overcome VEGF-targeted therapy resistance.

\section{MET}

The MET signaling pathway is important in tumor growth, survival, and metastasis and acts synergistically with VEGF to promote angiogenesis. ${ }^{33-35}$ In mouse models, the hepatocyte growth factor-MET pathway plays a key role in bone metastasis development. ${ }^{36}$ In renal cancer cells, MET protects cells from apoptosis, increases invasion and motility, and fosters epithelial-mesenchymal transition. ${ }^{37}$ As demonstrated in animal models and preclinical studies, inhibition of VEGF alone without MET produces rapid progression of tumors. ${ }^{27}$ Preclinical studies have shown that by blocking the VEGF pathway, hypoxia is induced, but the stress on the tumor cells results in a compensatory upregulation of MET expression, which further stimulates angiogenesis and migration away from the hypoxic zone..$^{38}$ After inhibition of cellular receptors of VEGF that promote angiogenesis, tumor cells attempt to escape the hypoxic environment by metastasizing and seeking a more hospitable location. This rapid revascularization and subsequent metastasis is presaged by MET overexpression, leads to resistance of the VEGF pathway, and is a fundamental mechanism that tumor cells use to survive. In in vivo studies of sunitinib, the MET pathway was identified as important in the development of resistance to VEGF signaling inhibition. In the experimental model, flow cytometry showed that MET expression was higher in resistant tumor cells. Combining sunitinib with a selective MET inhibitor significantly enhanced tumor suppression when compared with sunitinib or MET inhibitor alone in resistant tumors. ${ }^{39}$

The discovery that VEGFR and MET pathways work synergistically in tumor cells underlies the development of cabozantinib. ${ }^{40-42}$ Similar to other VEGF TKIs, cabozantinib inhibits vascular endothelial cell tubule formation in vitro, cellular migration, invasion, and tumor cell proliferation in a variety of tumor types. Uniquely, cabozantinib inhibits MET and VEGFR2 phosphorylation in vivo, preventing compensatory metastasis following intravenous tumor cell inoculation. By targeting both pathways simultaneously, cabozantinib blocks metastatic escape pathways and inhibits the expression of other potential resistance factors such as phosphatidylinositol-3-kinase, phospholipase C, pp60 ${ }^{\text {src }}$, and Grb2/Sos $1{ }^{43}$ Preclinical studies found that cabozantinib inhibited the appearance of liver metastasis in a transgenic mouse xenograft model. ${ }^{44}$ Treatment with cabozantinib resulted in a more extensive tumor shrinkage and a decreased tumor invasiveness and metastasis than treatment with vehicle or anti-VEGF antibody alone. The median survival

\section{Upregulation of MET as a response to treatment}
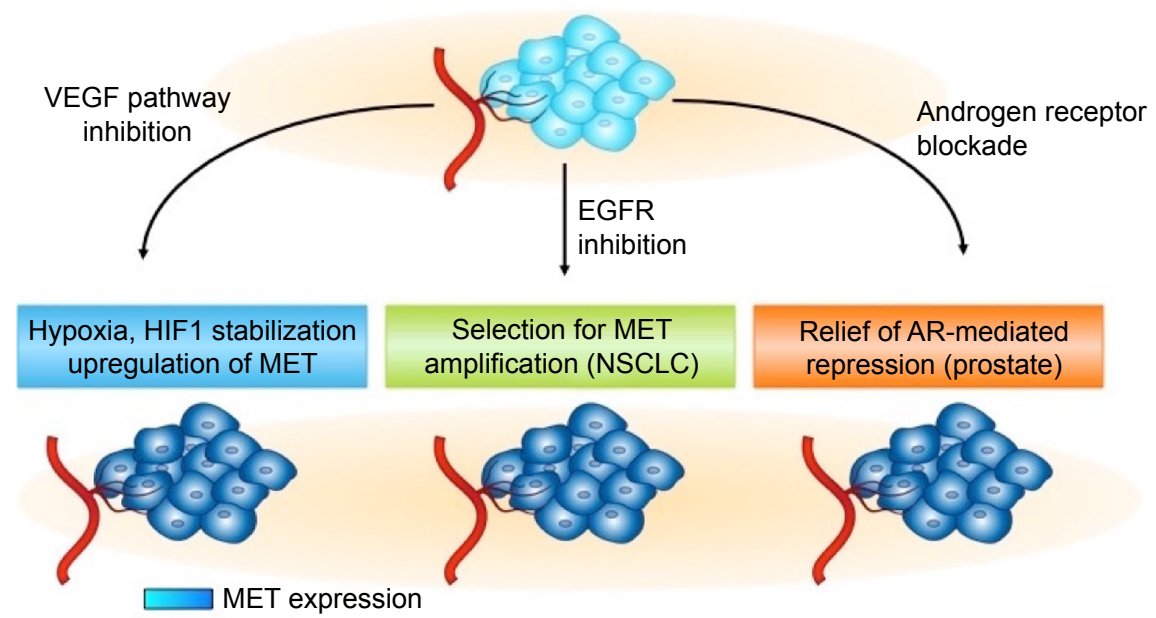

Figure 2 VEGF, HIFI, and MET are upregulated in hypoxic environments to promote survival by stimulating angiogenesis or facilitating migration away from the hypoxic zone. Note: Adapted with permission from Exelixis, Inc. (C) 2016..$^{85}$

Abbreviations: AR, androgen receptor; NSCLC, non-small cell lung cancer. 
was 14.7 weeks for vehicle-treated animals and 16.4 weeks for anti-VEGF antibody-treated animals, while animals treated with MET inhibitors demonstrated an improved survival of $>20$ weeks $(P<0.05)$. Of significance in the study, all of the mice treated with cabozantinib survived until the experiment ended at 20 weeks, whereas none of the vehicle- or anti-VEGF antibody-treated mice survived to that endpoint. ${ }^{45}$ This study suggests that dual inhibition of MET and VEGFR2 with cabozantinib is more effective and results in a more sustained response than inhibition of VEGF pathway alone.

\section{Role of cabozantinib}

In 2011, cabozantinib emerged as a new, orally bioavailable, small-molecule receptor TKI with dual VEGF and MET inhibition properties. Preclinical experiments found that inhibition of MET and VEGFR2 phosphorylation in vitro and in tumor models in vivo led to significant reductions in cell invasion. In vivo, the effect of disruption of tumor vasculature by cabozantinib was studied on antiangiogenicsensitive tumor cells expressing MET and VEGF in animal models. ${ }^{44,46-49}$ The animals were divided into two groups in which one group was administered the drug with vehicle and the second group was administered only the drug. Both the groups were administered the same dose orally, and after first 4 and 8 hours intervals, tumors were collected. Consecutive tumor collection was done after 4 hours interval following second, third, and fourth administrations of the drug. Cabozantinib-treated tumor cells exhibited $\sim 13$ times greater levels of tumor hypoxia and 2.5 times greater levels of apoptosis, measured by cell death marker TUNEL, after the first and second doses as compared to the vehicletreated tumors. ${ }^{25}$ In mouse models, cabozantinib dramatically altered tumor pathology, resulting in decreased tumor and endothelial cell proliferation coupled with increased apoptosis and dose-dependent inhibition of tumor growth in various tumors. Cabozantinib was also found to bind to and inhibit other tyrosine kinases implicated in tumor pathobiology involved in angiogenesis including RET, KIT, AXL, and FLT3 (Figure 3). ${ }^{50,51}$

A Phase I study was undertaken using cabozantinib in a dose de-escalation design beginning at $140 \mathrm{mg}$ orally daily in 25 heavily pretreated patients with metastatic renal cell cancer. ${ }^{52}$ The most common clinical side effects were fatigue (80\%), diarrhea (64\%), anorexia (36\%), and vomiting (36\%). Common grade $\geq 3$ adverse events included fatigue (20\%), diarrhea $(12 \%)$, pulmonary embolism (12\%), hypophosphatemia (40\%), proteinuria (8\%), appetite decreased (4\%), palmar-plantar erythrodysesthesia (4\%), and vomiting (4\%). Dose reductions occurred in 20 of the 25 patients: the final daily dose was $100 \mathrm{mg}$ for 6 patients, $60 \mathrm{mg}$ for 11 patients, $40 \mathrm{mg}$ for 2 patients, and $20 \mathrm{mg}$ for 1 patient. The median average daily dose was $75.5 \mathrm{mg}$. Of the 21 subjects assessable for radiological response by RECIST, partial response was reported in 7 patients (28\%) while 13 patients $(52 \%)$ had stable disease and only 1 patient had progressive disease as the best response. The median PFS was 12.9 months, and the median overall survival was 15.0 months. At the conclusion of this trial, it was clear that cabozantinib promised significant efficacy compared to historical controls for cancer control and PFS and overall survival. Questions remained regarding toxicity; therefore, the optimal dose in renal cell cancer was decided based on experiences in other solid tumor studies. ${ }^{53-57}$

With robust Phase I evidence of cabozantinib activity in RCC, researchers embarked on a phase III trial, known as the METEOR trial, without conducting intercurrrent Phase II trials. ${ }^{58}$ The METEOR trial compared cabozantinib with everolimus in 658 patients with RCC who had experienced disease progression following treatment with a VEGF receptor

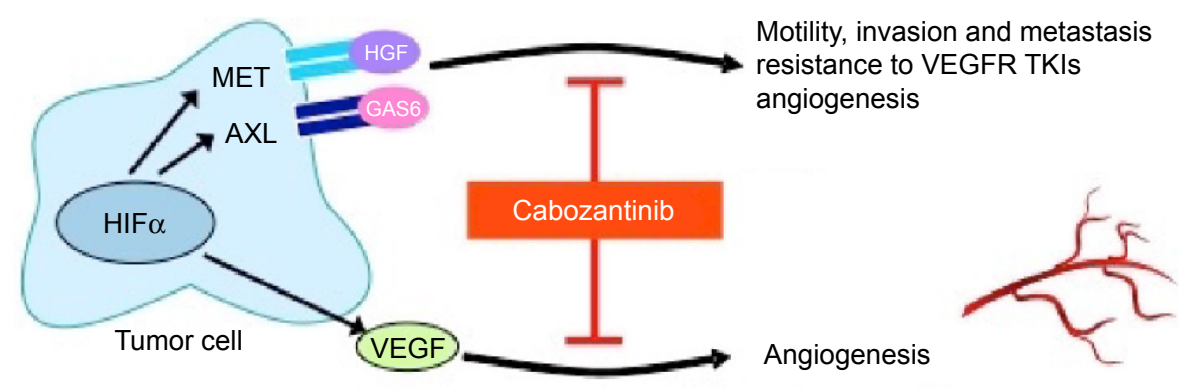

Figure 3 Cabozantinib binds to and inhibits tyrosine kinases implicated in tumor pathobiology involved in angiogenesis including VEGF, MET, and AXL to block motility, invasion, metastasis, and angiogenesis.

Note: Adapted with permission from Exelixis, Inc. (C) 2016. ${ }^{85}$

Abbreviation: TKIs, tyrosine kinase inhibitors. 
TKI. Patient selection required histological or cytological diagnosis of renal cell cancer with a clear-cell component, measurable disease, recovery from prior toxicities related to any prior treatments. 658 patients were randomized in a 1:1 ratio to receive cabozantinib at a starting dose of $60 \mathrm{mg}$ daily (330 patients) or everolimus (328 patients). The median age of patients was $\sim 62$ years (range, $31-86$ years). All patients had received at least one prior VEGFR-targeted TKI and had radiographic progression within 6 months after the most recent dose. Previous systemic therapy primarily consisted of sunitinib (62\%), pazopanib (43\%), and axitinib (16\%). The primary endpoint of PFS was assessed on the first 375 patients enrolled in the trial. In this portion of the study, 187 patients were randomized to cabozantinib and 188 received everolimus. The median PFS almost doubled from 3.8 months (95\% CI 3.7-5.4) with everolimus to 7.4 months (95\% CI: 5.6-9.1) with cabozantinib, representing a $42 \%$ reduction in the risk of progression or death $(P<0.001)$. Cabozantinib was superior to everolimus for PFS across all subgroups. Objective tumor responses were observed in $21 \%$ of the cabozantinib group compared to $5 \%$ of patients treated with everolimus. When further assessed by an independent radiology process, the median PFS across all enrolled patients was 7.4 months for the cabozantinib arm vs 3.9 months for the everolimus arm, corresponding to a $48 \%$ reduction in the rate of disease progression or death for cabozantinib as compared to everolimus (HR $=0.52,95 \%$ CI $0.43-0.64, P<0.001)$. The objective response rate was $17 \%$ for cabozantinib and $3 \%$ for everolimus. In the secondary endpoint of the trial, the entire study population of 658 patients showed a median overall survival of 21.4 months (95\% CI: 18.7-not estimable) with cabozantinib and 16.5 months (95\% CI: 14.7-18.8) with everolimus (HR $=0.66,95 \%$ CI $0.53-0.83 ; P=0.00026)$. The pivotal results of the METEOR trial led to the approval of cabozantinib by the FDA for the treatment of advanced RCC in 2016.

\section{Dosing and toxicity I $40 \mathrm{mg}$}

Initial studies showed that cabozantinib inhibits tumor growth in a dose-dependent manner, and dose escalation was evaluated. The maximum tolerated dose in the Phase I study specifically in medullary thyroid cancer patients was $175 \mathrm{mg}$ daily with dose-limiting grade 3 or worse toxicities including fatigue (10\%), elevation of lipase (9\%), and amylase $(5 \%)$, as well as diarrhea, weight loss, and transaminitis. For medullary thyroid cancer, a dose of $140 \mathrm{mg}$ daily was chosen for the registration trial and became the FDA-approved dose for this indication.
A follow-up Phase III trial, EXAM, demonstrated substantial PFS prolongation with cabozantinib vs placebo in patients with medullary thyroid cancer. A total of 330 patients were administered cabozantinib in a 2:1 ratio either in $140 \mathrm{mg}$ base form (219 patients) or placebo (111 patients). The most common grade $3 / 4$ adverse events were diarrhea (cabozantinib $15.9 \%$ vs placebo $1.8 \%$ ), hand-foot syndrome (cabozantinib $12.6 \%$ vs placebo $0 \%$ ), fatigue (cabozantinib $9.3 \%$ vs placebo $2.8 \%$ ), hypocalcemia (cabozantinib 9.3\% vs placebo $0 \%$ ), and hypertension (cabozantinib $7.9 \%$ vs placebo $0 \%$ ). Notably, dose reductions occurred in $79 \%$ of patients and dose interruptions occurred in $65 \%$ patients in the cabozantinib arm. As a result of adverse events, $16 \%$ of patients treated with cabozantinib had the drug discontinued. ${ }^{59}$ The need for monitoring the adverse events on cabozantinib is supported by a recent meta-analysis of 22 publications looking at response and toxicity of TKIs in patients with thyroid carcinoma. In this meta-analysis, cabozantinib at $140 \mathrm{mg}$ was found to be associated with the highest percentage of dose reductions and/or discontinuation among TKIs. ${ }^{60}$

Based on dosing used to treat medullary thyroid cancer, a Phase I trial on RCC was conducted with a dose of $140 \mathrm{mg}$ of cabozantinib daily. The trial showed encouraging efficacy but considerable toxicity as previously mentioned. Comparable to the medullary thyroid cancer studies, treatment discontinuation occurred in $24 \%$ of patients due to adverse events. Dose reductions occurred in $80 \%$ of patients. The median average daily dose was $75.5 \mathrm{mg}$ cabozantinib (range, $43.8-137.5 \mathrm{mg}$ ), and the median dose intensity percentage was $53.9 \%$ (range, $31.3 \%-98.2 \%$ ). ${ }^{53}$

\section{$100 \mathrm{mg}$}

Due to the high toxicities seen in the $140 \mathrm{mg}$ dose of cabozantinib, subsequent Phase II trials were conducted on lower doses of cabozantinib in other tumor types, including metastatic prostate cancer. In a Phase II randomized discontinuation study, 171 patients with metastatic castrate-resistant prostate cancer received cabozantinib $100 \mathrm{mg}$ daily: $68 \%$ of them had partial or complete resolution of bone scans and $67 \%$ of patients with pain at baseline reported a decrease in pain. However, by week 12 , dose reductions occurred in $51 \%$ of patients, with $12 \%$ discontinuing treatment due to adverse events. The most common grade 3 toxicities were fatigue, hypertension, and hand-foot syndrome. The most common serious adverse event was pulmonary embolism $(6 \%) .{ }^{61}$ A follow-up study with 144 patients with metastatic prostate cancer investigated cabozantinib at daily starting doses of $100 \mathrm{vs} 40 \mathrm{mg}$ until disease progression or 
Table I Summarizing key features of the METEOR trial

\begin{tabular}{llllllll}
\hline Therapy & $\begin{array}{l}\text { Rates of dose } \\
\text { reduction }\end{array}$ & $\begin{array}{l}\text { Median } \\
\text { average daily } \\
\text { dose } \mathbf{( m g )}\end{array}$ & $\begin{array}{l}\text { Rate of } \\
\text { discontinuation }\end{array}$ & $\begin{array}{l}\text { Grade 3/4 } \\
\text { adverse } \\
\text { events }\end{array}$ & ORR $^{\text {b }}$ & $\begin{array}{l}\text { Median } \\
\text { PFS (months) }\end{array}$ & $\begin{array}{l}\text { OS (months) } \\
\text { Cabozantinib N=331 }\end{array}$ \\
\hline Everolimus N=322 & $25 \%(197)$ & 44 & $12 \%(40)$ & $68 \%(226)$ & $21 \%(40)$ & 7.4 & 21.4 \\
\hline
\end{tabular}

Notes: ${ }^{\mathrm{T}}$ The rate of treatment discontinuation due to adverse events not related to renal cell carcinoma in the cabozantinib group and in the everolimus group. ${ }^{\mathrm{b}} \mathrm{Among}$ the first 375 patients who underwent randomization 187 patients assigned to cabozantinib and I88 patients assigned to everolimus. Data from Choueiri et al. ${ }^{58}$

Abbreviations: PFS, progression-free survival; ORR, overall response rate; OS, overall survival.

unacceptable toxicity. Though there was bone scan response and clinically meaningful pain relief in the majority of patients, in the $100 \mathrm{mg}$ cohort, $84 \%$ of patients had at least one dose reduction and $25 \%$ discontinued treatment because of an adverse event. In the $40 \mathrm{mg}$ cohort, $31 \%$ of patients had at least one dose reduction because of an adverse event and $18 \%$ of them discontinued treatment. Interestingly, the median average daily dose received in the $100 \mathrm{mg}$ and $40 \mathrm{mg}$ cohorts was 55 and $36 \mathrm{mg} / \mathrm{d}$, respectively. As a result, the starting doses of $\leq 100 \mathrm{mg}$ were thought to be advisable for follow-up studies due to tolerability concerns while having equivalent efficacy as the true difference in actual dose administered between the cohorts was not large. ${ }^{62}$

\section{$60 \mathrm{mg}$}

Subsequent studies in prostate cancer, the COMET program, utilized $60 \mathrm{mg}$ as the starting dose. Phase II studies conducted on lower doses of 60 and $40 \mathrm{mg}$ of cabozantinib resulted in less adverse events and continued clinical efficacy. ${ }^{63}$ COMET 1 and 2 were randomized, double blind, controlled trials completed for metastatic castrate-resistant prostate cancer at a starting dose of cabozantinib of $60 \mathrm{mg}$ daily. Sixty percent of patients who received cabozantinib experienced at least one dose reduction. ${ }^{56}$ COMET 2 compared cabozantinib with mitoxantrone and prednisone (MP): $55 \%$ of the patients receiving cabozantinib experienced at least one dose reduction compared to $14 \%$ of the patients receiving MP. Seventy percent of the patients in the cabozantinib group had a grade $3 / 4$ adverse event. However, fewer patients discontinued study treatment for adverse events in the cabozantinib $\operatorname{arm}\left(16 \%\right.$ vs $26 \%$ for MP). ${ }^{57}$

In the METEOR trial, the $60 \mathrm{mg}$ cabozantinib dose was used as the starting dose; notably dose reductions were still required in $60 \%$ of patients in the cabozantinib arm, most commonly due to diarrhea, plantar palmar dysesthesia, hypertension, and fatigue. The median average daily dose was $44 \mathrm{mg}$ for cabozantinib and $9 \mathrm{mg}$ for everolimus. Serious adverse events occurred equally in both the cabozantinib and everolimus groups ( $40 \%$ and $43 \%$, respectively).
Treatment discontinuation because of an adverse event not related to disease progression was recorded in $40(12 \%)$ of 331 patients in the cabozantinib group and 34 (11\%) of 322 patients in the everolimus group (Table 1). Death considered related to study treatment occurred in one patient in the cabozantinib group (death not otherwise specified) and in two patients in the everolimus group (aspergillosis and aspiration pneumonia)..$^{58}$

Importantly, cabozantinib at lower doses continues to have clinical efficacy, especially in medullary thyroid cancer and RCC with much improved tolerability. Previous metaanalysis showing cabozantinib to have a highest percentage of discontinuation among TKIs was studied with a starting dose of $140 \mathrm{mg}$ much higher than the approved $60 \mathrm{mg}$ dose in RCC.$^{60}$ When comparing across trials, the $60 \mathrm{mg}$ dose has a similar discontinuation rate to sorafinib, which is lower than other TKIs (Table 2). The median average daily dose in the METEOR trial was $43 \mathrm{mg}$ for cabozantinib and suggests that the toxicity threshold may be slightly $>40 \mathrm{mg}$ rather than the starting dose of $60 \mathrm{mg}$. Analysis of the CABOSUN study, where cabozantinib is being compared with sunitinib for previously untreated patients with advanced intermediate- or poor-risk RCC renal cancer, will provide more data on comparative toxicity and tolerability and also raise the possibility

Table 2 Summary of selected Phase III trials of advanced RCC therapeutics and dose discontinuations due to adverse events

\begin{tabular}{llll}
\hline Trial & $\begin{array}{l}\text { Systemic } \\
\text { therapy }\end{array}$ & N & $\begin{array}{l}\text { Dose } \\
\text { discontinuation (\%) }\end{array}$ \\
\hline Rini et al ${ }^{12}$ & Sorafenib & 355 & 8 \\
Sternberg et al & Axitinib & 359 & 4 \\
& Pazopanib & 290 & 14 \\
Motzer et al $^{14}$ & Placebo & 145 & 3 \\
& Pazopanib & 554 & 24 \\
Choueiri et al $^{58}$ & Sunitinib & 548 & 20 \\
& Cabozantinib & 331 & 12 \\
Motzer et al & Everolimus & 322 & 11 \\
& Nivolumab & 406 & 8 \\
Hudes et al & Everolimus & 397 & 13 \\
& Temsirolimus & 209 & 15 \\
& Interferon alpha & 207 & 29 \\
\hline
\end{tabular}

Abbreviation: RCC, renal cell carcinoma. 
Table 3 Ongoing trials with cabozantinib alone or in combination for metastic RCC

\begin{tabular}{|c|c|c|c|c|c|}
\hline $\begin{array}{l}\text { ClinicalTrials.gov } \\
\text { identifier }\end{array}$ & Arms & $\begin{array}{l}\text { Line of } \\
\text { therapy }\end{array}$ & $\mathbf{N}$ & Starting dose & Primary endpoint \\
\hline $\begin{array}{l}\text { NCTOI835I5864 } \\
\text { Phase II (CABOSUN) }\end{array}$ & $\begin{array}{l}\text { Cabozantinib } \\
\text { Sunitinib }\end{array}$ & $\begin{array}{l}\text { First-line } \\
\text { metastatic }\end{array}$ & 150 & $\begin{array}{l}\text { Cabozantinib } 60 \mathrm{mg} \text { daily } \\
\text { Sunitinib } 50 \mathrm{mg} \text { ( } 4 \text { weeks } \\
\text { on and } 2 \text { weeks off) }\end{array}$ & OS and PFS ( 5 years) \\
\hline $\begin{array}{l}\text { NCT02496208 } \\
\text { Phase I }\end{array}$ & $\begin{array}{l}\text { Cabozantinib + nivolumab } \\
\text { Cabozantinib + nivolumab + ipilimumab }\end{array}$ & $\begin{array}{l}\text { First-line } \\
\text { metastatic }\end{array}$ & 66 & $\begin{array}{l}\text { Cabozantinib } 60 \mathrm{mg} \text { with } \\
\text { dose escalation }\end{array}$ & $\begin{array}{l}\text { Determine the } \\
\text { dose-limiting toxicity }\end{array}$ \\
\hline $\begin{array}{l}\text { NCT0276I057 } \\
\text { Phase II (SWOG SI500) }\end{array}$ & $\begin{array}{l}\text { Cabozantinib Crizotinib } \\
\text { Sunitinib } \\
\text { Volitinib }\end{array}$ & $\begin{array}{l}\text { First-line } \\
\text { metastatic }\end{array}$ & 180 & $\begin{array}{l}\text { Cabozantinib } 60 \mathrm{mg} \text { daily } \\
\text { Crizotinib } 250 \mathrm{mg} \text { bid } \\
\text { Sunitinib } 50 \mathrm{mg} \text { daily } \\
\text { Volitinib } 600 \mathrm{mg} \text { daily }\end{array}$ & PFS \\
\hline
\end{tabular}

Abbreviations: PFS, progression-free survival; RCC, renal cell carcinoma; OS, overall survival.

of its use as a first-line drug. ${ }^{64}$ This trial and other ongoing trials are summarized in Table 3.

\section{Dose equivalence}

While cabozantinib in capsule formulation was approved for the treatment of medullary thyroid cancer (Cometriq), cabozantinib was reformulated into tablets with greater bioavailability, and this formulation (Cabometyx) was tested in the METEOR trial. When studying pharmacokinetics of cabozantinib tablet and capsule formulations in healthy adults, there are slight differences. A Phase I, randomized, open-label single-dose study in healthy individuals characterized the plasma pharmacokinetics of cabozantinib capsule, and tablet formulations showed a $19 \%$ increase in the $C_{\max }$ of the tablet formulation compared to the capsule formulation following a single $140 \mathrm{mg}$ dose. However, a $<10 \%$ difference in the AUC was observed between cabozantinib tablet and capsule formulations. Thus, in pharmacokinetic studies, the tablet and capsule formulations failed to fulfill the bioequivalence study acceptance criteria. ${ }^{65}$ Though not a large difference in pharmacokinetic activity, the results suggest that patients with RCC should only be given the tablet form (Cabometyx) to ensure more accurate monitoring and the tablet form is not interchangeable with the capsule form.

\section{Discussion}

The advent of VEGF and mTOR inhibitors in RCC has significantly improved the prognosis for patients with metastatic RCC. In patients not eligible for IFN $\alpha$ or interleukin-2, VEGF TKIs have replaced cytokines as the standard of care in metastatic RCC. However, complete responses are rare $(3 \%)$, and identifying agents effective in the setting of de novo or acquired resistance remains an important goal. ${ }^{10}$ The METEOR trial established cabozantinib as a choice for treatment in the second line and beyond, validating the preclinical work suggesting that MET acts as an alternative angiogenic pathway in the development of VEGF TKI resistance (Figure 4). As yet there are no known biomarkers to help with selection of patients for whom cabozantinib will be most effective; this has remained an elusive goal throughout the RCC therapeutic landscape.

Toxicity remains an important concern in the management of metastatic RCC, especially as more effective treatments result in longer durations of therapy. A meta-analysis recently drew attention to the high incidence and increased risk of developing hand-foot syndrome with cabozantinib, possibly in a dose-dependent manner. ${ }^{66}$ When comparing across trials, the approved starting dose of cabozantinb has similar and even less discontinuation due to adverse events compared to other TKIs. Proactive and aggressive management of this toxicity and others will be critical for maintaining the quality of life for RCC patients on cabozantinib. Dose reductions and discontinuations for the aforementioned trials are summarized in Tables 2 and 4.

\section{Future directions}

Further studies are needed to delineate the optimal cabozantinib dose. Patients should be counseled on its toxicity profile prior to treatment. As clinicians, we may need to reassess current dosing strategies and can look at other TKIs for prospective adjustments in dosing. A 2 weeks-on and 1 week-off schedule of sunitinib has shown decreased toxicity in metastatic RCC patients who initially experience grade $\geq 3$ toxicity on the $4 / 2$ schedule and a $2 / 1$ schedule can extend treatment duration considerably ${ }^{67}$ In the RAINBOW trial, a large retrospective analysis of 208 patients found that switching sunitinib to an alternate 2 weeks on and 1 week off schedule without affecting dose density over a 6-week cycle was associated with improved outcomes and increased 


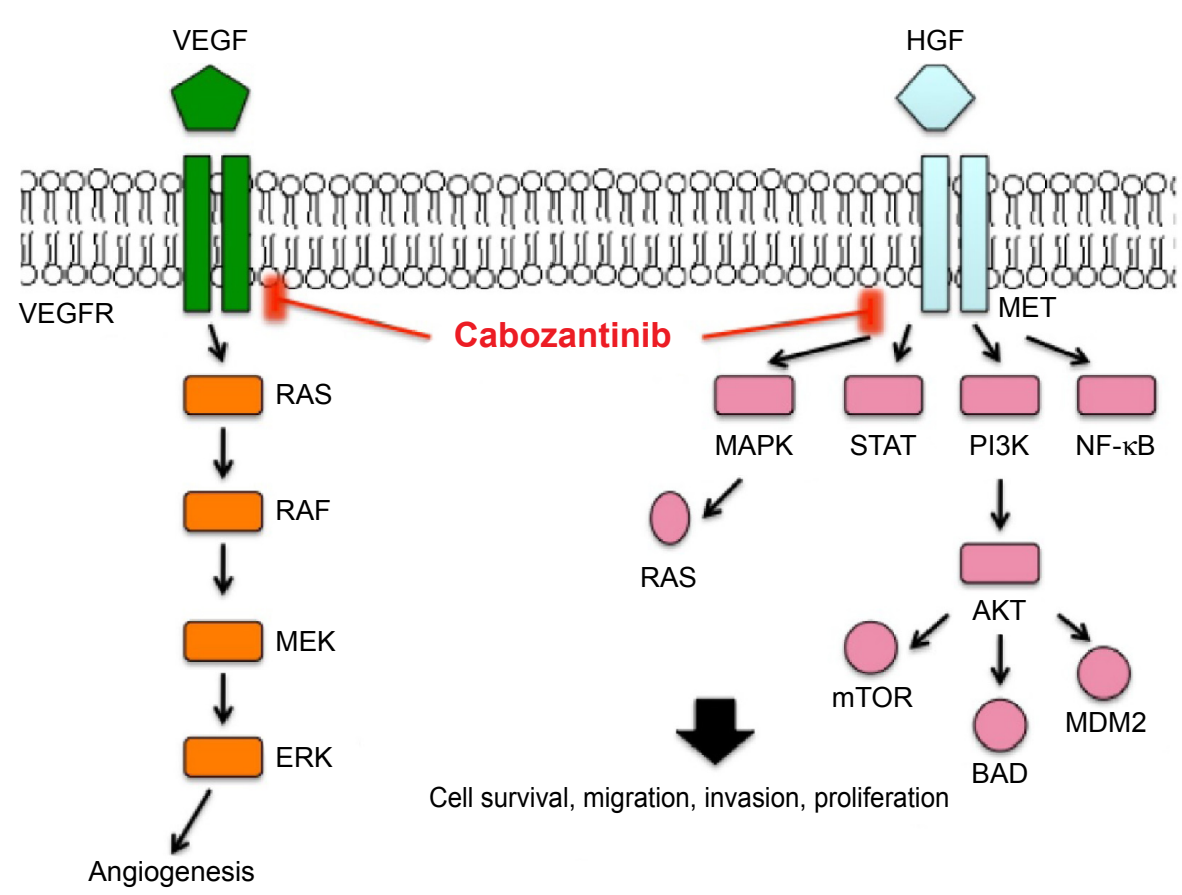

Figure 4 Cabozantinib provides dual inhibition of MET and VEGFR2 preventing the MET pathway from acting as an alternative pathway in the development of VEGF TKI resistance.

tolerability. The $2 / 1$ schedule reduced overall incidence of grade 3-4 toxicities to $8.2 \%$ compared to $45.7 \%$ in the $4 / 2$ schedule in the same patients and $29.4 \%$ in the control arm. ${ }^{68}$ Other studies have shown a lower incidence of dose interruption and similar outcomes compared with the standard dosing schedule of 4 weeks on and 2 weeks off. ${ }^{69-71}$

The sequence that cabozantinib should be used among the growing number of therapeutic options is also up for investigation. Though cabozantinib's approval was based on patients with advanced RCC who had received prior anti-angiogenic therapy, there may be a role for the drug in treatment-naïve patients as a first-line therapy. The Phase II CABOSUN study will compare cabozantinib to a $4 / 2$ schedule of sunitinib in the first-line setting and compare the tolerability of these regimens. Patients were randomized in a 1:1 ratio to $60 \mathrm{mg}$ once daily of cabozantinib or $50 \mathrm{mg}$ once daily ( 4 weeks on and 2 weeks off) of sunitinib with the primary outcome measured as PFS and OS, with the secondary endpoints including objective response rate. If the safety profile is similar to previously reported studies and shows improved PFS, OS, or ORR when compared with sunitinib, then cabozantinib could prove to be the new standard firstline therapy for untreated advanced RCC. ${ }^{64}$ In the secondline setting, cabozanitinib is considered a standard of care therapy in patients who have received prior antiangiogeneic therapy. Similar to other TKIs, cabozantinib can be given safely with careful adverse-event monitoring and appropriate dose reductions.

In addition to cabozantinib, nivolumab is an anti-PD-1 antibody newly approved as a second-line option for

Table 4 Summary of trial dose reductions and discontinuations

\begin{tabular}{|c|c|c|c|c|c|}
\hline Trial & $\begin{array}{l}\text { Cancer } \\
\text { type }\end{array}$ & $\begin{array}{l}\text { Cabozantinib } \\
\text { dose }(\mathrm{mg})\end{array}$ & $\mathbf{N}$ & $\begin{array}{l}\text { Dose } \\
\text { reduction (\%) }\end{array}$ & $\begin{array}{l}\text { Dose } \\
\text { discontinuation (\%) }\end{array}$ \\
\hline Choueiri et al ${ }^{52}$ & RCC & 140 & 25 & 80 & 24 \\
\hline Schoffski et al ${ }^{59}$ (EXAM) & MTC & 140 & 219 & 79 & 16 \\
\hline Smith et $a^{\mid 61}$ & Prostate $\mathrm{Ca}$ & 100 & $17 \mid$ & 51 & 12 \\
\hline \multirow[t]{2}{*}{ Leibowitz-Amit et al ${ }^{62}$} & Prostate Ca & 100 & 93 & 84 & 25 \\
\hline & & 40 & 51 & 31 & 18 \\
\hline Smith et al ${ }^{56}$ (COMET I) & Prostate $\mathrm{Ca}$ & 60 & 682 & 67 & 33 \\
\hline Basch et $\mathrm{al}^{57}$ (COMET 2) & Prostate & 60 & 61 & 55 & 16 \\
\hline Choueiri et al ${ }^{58}$ (METEOR trial) & $\mathrm{RCC}$ & 60 & 331 & 62 & 12 \\
\hline
\end{tabular}

Abbreviations: MTC, medullary thyroid cancer; RCC, renal cell carcinoma. 
metastatic RCC. Currently, there are no trials comparing cabozantinib with nivolumab, but an ongoing combination trial could prove to be beneficial. ${ }^{72}$ Nivolumab is an anti-PD-1 antibody that was initially developed for patients with advanced melanoma and non-small-cell lung cancer and has shown activity in advanced RCC. In a large Phase III trial, Checkmate-025, nivolumab prolonged overall survival when compared with everolimus as a second-line therapy in patients progressing after initial treatment with a VEGF TKI. The toxicity profile was notably low with grade 3 or 4 treatment-related adverse events occurring in only $19 \%$ of patients receiving nivolumab. ${ }^{73}$ Nivolumab competes with cabozantinib after progression on first-line agents or for patients unable to tolerate cabozantinib. While these drugs have distinct mechanisms of action, the combination of cabozantinib and nivolumab may also lead to a potential benefit of immune modulation in genitourinary tumors. In a study of metastatic urothelial cancer, regulatory $\mathrm{T}$ cell levels prior to cabozantinib treatment have been shown predictive of therapeutic responsiveness and overall survival. When assessing myeloid-derived suppressor cells and regulatory $\mathrm{T}$ cells in patients undergoing treatment with cabozantinib after 2 cycles, patients with low regulatory $T$ cells measured in peripheral blood samples at baseline had an improved response rate, PFS, and OS. Regulatory $\mathrm{T}$ cells decreased and $\mathrm{PD}-1$ expression in regulatory $\mathrm{T}$ cells increased after cabozantinib treatment ( $P=0.015$ and $P=0.011$, respectively). Patients with less change in PD-1 levels showed a trend to an improved PFS, and a decrease in myeloid-derived suppressor cells' expression on treatment was associated with an improved PFS. These results suggest that changes in regulatory $\mathrm{T}$ cell checkpoint molecule expression and myeloidderived suppressor cells expression may be prognostic and/ or predictive markers in patients with metastatic urothelial carcinoma treated with cabozantinib. ${ }^{74}$ Such results have led to investigation of a Phase I trial of cabozantinib and nivolumab with or without ipilimumab in treating patients with genitourinary tumors (NCT02496208). Currently, there are no other cabozantinib-based combination therapies under trial but this remains a tantalizing area of investigation.

Cabozantinib should also be compared with other secondline agents approved for RCC. Cabozantinib has yet to be compared with axitinib in clinical trials, and given that both agents are now approved in the second-line setting, this would have merit. Cabozanitinib should also be compared with the combination of levatinib + everolimus, which recently was shown to be superior to everolimus alone. ${ }^{75}$ An ongoing randomized Phase II trial comparing PFS with cabozantinib, crizotinib, volitinib, or sunitinib in metastatic papillary RCC will provide efficacy and toxicity data. ${ }^{76}$

There may also be a role of cabozantinib in the adjuvant setting. The ASSURE trial looked at 1,943 patients with resected, intermediate- or high-risk kidney cancer (both clear cell and nonclear cell) and randomly assigned them to 1 year of adjuvant sorafenib, sunitinib, or placebo. There were no significant differences in disease-free survival or overall survival between the drug and placebo arm. The median disease-free survival was 5.8 years in both the sorafenib and sunitinib arms and 6 years in the placebo arm. ${ }^{77}$ Though these results suggest a lack of benefit of TKIs in the adjuvant setting, the ongoing SORCE, S-TRAC, and ATLAS trials investigate the use of adjuvant sorafenib and axitinib for 3 years, respectively. ${ }^{78-80}$ If these studies prove positive, it raises the possibility that cabozantinb could also be beneficial in the adjuvant setting after surgery, radiofrequency ablation, or cryoablation.

Currently, there are no biomarkers related to cabozantinib in RCC, but such a discovery might improve the utility of cabozantinib. In prostate cancer, cabozantinib had some activity in reducing circulating tumor cells and bone biomarkers on treatment, but the drug did not impact PSA in the Phase III COMET 1 study. In an analysis of a Phase II trial, plasma concentrations of VEGFR2, Trap5b, Angiopoietin-2, TIMP-2, and TIE-2 significantly decreased during treatment with cabozantinib; there was no significant associations between any biomarker and type of response. ${ }^{62}$ Carbozantinib also inhibits RET, ROS1, NTRK, MET, and AXL, and there may be utility in investigating its role in specific cancer genotypes. A Phase II study in patients with advanced nonsmall cell lung cancer is currently investigating cabozantinib in patients whose tumors express changes in RET, ROS1, or NTRK fusion or increased MET or AXL activity. In lung cancer, RET, ROS1, or NTRK fusion or increased MET or AXL activity gene leads to increased cell growth. ${ }^{81}$ If effective in non-small-cell lung cancer, similar studies with specific genotypes may be warranted in RCC. The MET pathway has been studied in papillary RCC with patients harboring a germline MET mutation showing high response rate in a Phase II study. ${ }^{82}$ Some patients with chromosome 7 and/or c-MET local alterations have also been shown to respond very well to pathway blockade, offering a potential area of investigation. ${ }^{83}$ In addition, cabozantinib has effects on immune markers and may counteract tumor-induced immunosuppression, providing a rationale for combining cabozantinib with immunotherapeutic strategies. In a study characterizing the pharmacodynamic effect of cabozantinib, 
cabozantinib had an effect on PDL-1 and CTLA-4 expression on regulatory $\mathrm{T}$ cells, and $\mathrm{T}$ regulatory cell levels may be future prognostic markers. ${ }^{84}$ If such biomarkers could be validated in RCC and followed throughout treatment, it may facilitate dynamic targeted intervention in advanced RCC.

\section{Disclosure}

SSY declares that he has no relevant conflicts of interest that relate to the research described in this paper. DIQ declares that he has conflicts of interest including advisory board position and speaker honorarium with Pfizer, Bayer, Novartis, Exelixis, BMS, and Argos. TBD declares that she has conflicts of interest including speaker honorarium with Astellas, Bayer, Dendreon, Exelixis, Genentech, and Pfizer. The authors report no other conflicts of interest in this work.

\section{References}

1. Ferlay J, Soerjomataram I, Ervik M, et al. [homepage on the Internet]. GLOBOCAN 2012 v1.1, Cancer Incidence and Mortality Worldwide: IARC CancerBase No 11 [Internet]. Lyon, France: International Agency for Research on Cancer; 2014. Available from: http://globocan.iarc.fr. Accessed January 16, 2015.

2. Torre LA, Bray F, Siegel RL, Ferlay J, Lortet-Tieulent J, Jemal A. Global cancer statistics, 2012. CA Cancer J Clin. 2015;65(2):87-108.

3. Cancer.net [webpage on the Internet]. Kidney cancer. Available from: http://www.cancer.net/patient/Cancer+Types/Kidney+Cancer? sectionTitle $=$ Risk $\% 20$ Factors $\% 20$ and $\% 20$ Prevention. Accessed July 17, 2012.

4. American Cancer Society. Detailed Guide: Kidney Cancer. (Adult) Renal Cell Carcinoma. 2012. Available from: http://www.cancer. org/acs/groups/cid/documents/webcontent/003107-pdf. Accessed July 17, 2012.

5. Siegel RL, Miller KD, Jemal A. Cancer statistics, 2015. CA Cancer J Clin. 2015;65(1):5.

6. National Cancer Institute [webpage on the Internet]. SEER Stat Fact Sheets: Kidney and Renal Pelvis. 2012. Available from: http://seer. cancer.gov/statfacts/html/kidrp.html. Accessed July 17, 2012.

7. Fyfe G, Fisher RI, Rosenberg SA, Sznol M, Parkinson DR, Louie AC. Results of treatment of 255 patients with metastatic renal cell carcinoma who received high-dose recombinant interleukin-2 therapy. $J$ Clin Oncol. 1995;13(3):688-696.

8. Heng DY, Xie W, Regan MM, et al. Prognostic factors for overall survival in patients with metastatic renal cell carcinoma treated with vascular endothelial growth factor-targeted agents: results from a large, multicenter study. J Clin Oncol. 2009;27(34):5794-5799.

9. Motzer RJ, Hutson TE, Tomczak P, et al. Sunitinib versus interferon alfa in metastatic renal-cell carcinoma. $N$ Engl J Med. 2007;356(2):115.

10. Motzer RJ, Hutson TE, Tomczak P, et al. Overall survival and updated results for sunitinib compared with interferon alfa in patients with metastatic renal cell carcinoma. J Clin Oncol. 2009;27(22):3584.

11. Patil S, Figlin RA, Hutson TE, et al. Prognostic factors for progressionfree and overall survival with sunitinib targeted therapy and with cytokine as first-line therapy in patients with metastatic renal cell carcinoma. Ann Oncol. 2011;22(2):295.

12. Rini BI, Escudier B, Tomczak P, et al. Comparative effectiveness of axitinib versus sorafenib in advanced renal cell carcinoma (AXIS): a randomised phase 3 trial. Lancet. 2011;378(9807):1931-1939.

13. Sternberg CN, Davis ID, Mardiak J, et al. Pazopanib in locally advanced or metastatic renal cell carcinoma: results of a randomized phase III trial. J Clin Oncol. 2010;28(6):1061.
14. Motzer RJ, Hutson TE, McCann L, Deen K, Choueiri TK. Overall survival in renal-cell carcinoma with pazopanib versus sunitinib. $N$ Engl J Med. 2014;370(18):1769.

15. Rini BI, Halabi S, Rosenberg JE, et al. Phase III trial of bevacizumab plus interferon alfa versus interferon alfa monotherapy in patients with metastatic renal cell carcinoma: final results of CALGB 90206. J Clin Oncol. 2010;28(13):2137.

16. Escudier B, Pluzanska A, Koralewski P, et al. Bevacizumab plus interferon alfa-2a for treatment of metastatic renal cell carcinoma: a randomised, double-blind phase III trial. Lancet. 2007;370(9605): 2103-2111.

17. Pantuck AJ, Seligson DB, Klatte T, et al. Prognostic relevance of the mTOR pathway in renal cell carcinoma. Cancer. 2007;109:2257-2267.

18. Hudes G, Carducci M, Tomczak P, et al. Temsirolimus, interferon alfa, or both for advanced renal-cell carcinoma. Global ARCC trial. $N$ Engl J Med. 2007;356(22):2271.

19. Hutson TE, Escudier B, Esteban E, et al. Randomized phase III trial of temsirolimus versus sorafenib as second-line therapy after sunitinib in patients with metastatic renal cell carcinoma. Clin Oncol. 2014;32(8):760-767.

20. Motzer RJ, Escudier B, Oudard S, et al. Phase 3 trial of everolimus for metastatic renal cell carcinoma: final results and analysis of prognostic factors. Cancer. 2010;116(18):4256-4265.

21. Hanahan D, Weinberg RA. The hallmarks of cancer. Cell. 2000;100(1): $57-70$.

22. Chung AS, Lee J, Ferrara N. Targeting the tumour vasculature: insights from physiological angiogenesis. Nat Rev Cancer. 2010;10(7):505.

23. Ferrara N, Gerber HP, LeCouter J. The biology of VEGF and its receptors. Nat Med. 2003;9(6):669.

24. Homsi J, Daud AI. Spectrum of activity and mechanism of action of VEGF/PDGF inhibitors. Cancer Control. 2007;14:285-294.

25. Yakes FM, Chen J, Tan J, et al. Cabozantinib (XL184), a novel MET and VEGFR2 inhibitor, simultaneously suppresses metastasis, angiogenesis, and tumor growth. Mol Cancer Ther. 2011;10:2298-2308.

26. Sharma T, Dhingra R, Singh S, et al. Aflibercept: a novel VEGF targeted agent to explore the future perspectives of anti-angiogenic therapy for the treatment of multiple tumors. Mini-Rev Med Chem. 2013; 13:530-540.

27. Pàez-Ribes M, Allen E, Hudock J, et al. Antiangiogenic therapy elicits malignant progression of tumors to increased local invasion and distant metastasis. Cancer Cell. 2009;15(3):220-231.

28. Bielecka ZF, Czarnecka AM, Solarek W, Kornakiewicz A, Szczylik C. Mechanisms of acquired resistance to tyrosine kinase inhibitors in clear - cell renal cell carcinoma (ccRCC). Curr Signal Transduct Ther. 2014;8(3):218-228.

29. Sierra JR, Tsao MS. c-MET as a potential therapeutic target and biomarker in cancer. Ther Adv Med Oncol. 2011;3(1 suppl):S21-S35.

30. Sennino B, Naylor R, Tabruyn S, et al. Abstract A13: reduction of tumor invasiveness and metastasis and prolongation of survival of RIP-Tag2 mice after inhibition of VEGFR plus c-Met by XL184 - Sennino et al. 8 (1001): A13 - molecular cancer therapeutics. Mol Cancer Ther. 2009;8:A13.

31. Zhang Y, Guessous F, Kofman A, Schiff D, Abounader R. XL-184, a MET, VEGFR-2 and RET kinase inhibitor for the treatment of thyroid cancer, glioblastoma multiforme and NSCLC. IDrugs. 2010;13(2):112-124.

32. You WK, Sennino B, Williamson CW, et al. VEGF and c-Met blockade amplify angiogenesis inhibition in pancreatic islet cancer. Cancer Res. 2011;71(14):4758-4768.

33. Miyata Y, Asai A, Mitsunari K, et al. Met in urological cancers. Cancers (Basel). 2014;6(4):2387-2403.

34. Xin X, Yang S, Ingle G, et al. Hepatocyte growth factor enhances vascular endothelial growth factor-induced angiogenesis in vitro and in vivo. Am J Pathol. 2001;158:1111-1120.

35. Van Belle E, Witzenbichler B, Chen D, et al. Potentiated angiogenic effect of scatter factor/hepatocyte growth factor via induction of vascular endothelial growth factor: the case for paracrine amplification of angiogenesis. Circulation. 1998;97:381-390. 
36. Ono K, Kamiya S, Akatsu T, et al. Involvement of hepatocyte growth factor in the development of bone metastasis of a mouse mammary cancer cell line BALB/c-MC. Bone. 2006;39(1):27-34.

37. Vaishampayan U. Cabozantinib as a novel therapy for renal cell carcinoma. Curr Oncol Rep. 2013;15(2):76-82.

38. Casanovas O, Hicklin DJ, Bergers G, Hanahan D. Drug resistance by evasion of antiangiogenic targeting of VEGF signaling in late-stage pancreatic islet tumors. Cancer Cell. 2005;8(4):299-309.

39. Shojaei F, Lee JH, Simmons BH, et al. HGF/c-Met acts as an alternative angiogenic pathway in suni-tinib-resistant tumors. Cancer Res. 2010;70:10090-10100.

40. Ebos JM, Lee CR, Cruz-Munoz W, Bjarnason GA, Christensen JG, Kerbel RS. Accelerated metastasis after short-term treatment with a potent inhibitor of tumor angiogenesis. Cancer Cell. 2009;15:232-239.

41. Di Tomaso E, Snuderl M, Kamoun WS, et al. Glioblastoma recurrence after cediranib therapy in patients: lack of "rebound" revascularization as mode of escape. Cancer Res. 2011;71:19-28.

42. Schmidt L, Junker K, Nakaigawa N, et al. Novel mutations of the MET proto-oncogene in papillary renal carcinomas. Oncogene. 1999;18: 2343-2350.

43. Trusolino L, Pugliese L, Comoglio PM. Interactions between scatter factors and their receptors: hints for therapeutic applications. FASEBJ. 1998; 12:1267-1280.

44. Sun Y, Sun L, An Y, Shen X. Cabozantinib, a novel c-met inhibitor, inhibits colorectal cancer development in a xenograft model. Med Sci Monit. 2015;21:2316-2321.

45. Sennino B, Ishiguro-Oonuma T, Wei Y, et al. Suppression of tumor invasion and metastasis by concurrent inhibition of c-Met and VEGF signaling in pancreatic neuroendocrine tumors. Cancer Discov. 2012; 2(3):270-287.

46. Navis AC, Bourgonje A, Wesseling P, et al. Effects of dual targeting of tumor cells and stroma in human glioblastoma xenografts with a tyrosine kinase inhibitor against c-MET and VEGFR2. PLoS One. 2013;8(3).

47. Torres KE, Zhu QS, Bill K, et al. Activated MET is a molecular prognosticator and potential therapeutic target for malignant peripheral nerve sheath tumors. Clin Cancer Res. 2011;17:3943-3955.

48. Roland CL, Dineen SP, Lynn KD, et al. Inhibition of vascular endothelial growth factor reduces angiogenesis and modulates immune cell infiltration of orthotopic breast cancer xenografts. Mol Cancer Ther. 2009;8:1761-1771.

49. Munshi N, Jeay S, Li Y, et al. ARQ 197, a novel and selective inhibitor of the human c-Met receptor tyrosine kinase with antitumor activity. Mol Cancer Ther. 2010;9:1544-1553.

50. Castellone MD, Carlomagno F, Salvatore G, Santoro M. Receptor tyrosine kinase inhibitors in thyroid cancer. Best Pract Res Clin Endo-Crinol Metab. 2008;22:1023-1038.

51. Timar J, Dome B. Antiangiogenic drugs and tyrosine kinases. AntiCancer Agents Med Chem. 2008;8:462-469.

52. Choueiri TK, Pal SK, McDermott DF, et al. A phase I study of cabozantinib (XL184) in patients with renal cell cancer. Ann Oncol. 2014; 25(8):1603-1608.

53. Durante C, Russo D, Verrienti A, Filetti S. XL184 (cabozantinib) for medullary thyroid carcinoma. Expert Opin Investig Drugs. 2011;20(3): 407-413.

54. Elisei R, Schlumberger MJ, Müller SP, et al. Cabozantinib in progressive medullary thyroid cancer. J Clin Oncol. 2013;31(29):3639-3646.

55. Hussain M, Smith MR, Sweeney C, et al. Cabozantinib (XL184) in metastatic castration-resistant prostate cancer (mCRPC): results from a phase II randomized discontinuation trial. 2011 ASCO Annual Meeting Abstracts Part 1. 2011:4516. J Clin Oncol. 29, No 15_suppl (May 20 Supplement).

56. Smith MR, De Bono JS, Sternberg CN, et al. Final analysis of COMET-1: cabozantinib versus prednisone in metastatic castrationresistant prostate cancer patients previously treated with docetaxel and abiraterone and/or enzalutamide. Proceedings of the 2015 Genitourinary Cancers Symposium. Presented February 26, 2015. Chicago, Illinois: 2015. J Clin Oncol. 33, 2015 (suppl 7; abstr 139).
57. Basch EM, Scholz MC, De Bono JS, et al. Final analysis of COMET-2: Cabozantinib (Cabo) versus mitoxantrone/prednisone (MP) in metastatic castration-resistant prostate cancer (mCRPC) patients (pts) with moderate to severe pain who were previously treated with docetaxel (D) and abiraterone (A) and/or enzalutamide (E). In: J Clin Oncol. 2015 Genitourinary Cancers Symposium; February 26-28, 2015. Orlando, Florida. 2015;33(Supp1 7), abstract 14.

58. Choueiri TK, Escudier B, Powles T, et al; METEOR Investigators. Cabozantinib versus everolimus in advanced renal cell carcinoma (METEOR): final results from a randomised, open-label, phase 3 trial. Lancet Oncol. 2016;17(7):917-927.

59. Schoffski P, Elisei R, Muller S, Brose MS, Shah MH, Licitra LF. In an international, double-blind, randomized, placebo-controlled phase III trial (EXAM) of cabozantinib (XL184) in medullary thyroid cancer (MTC) patients (pts) with documented RECIST progression at baseline. J Clin Oncol. 2012;30:5508.

60. Klein Hesselink EN, Steenvoorden D, Kapiteijn E, et al. Therapy of endocrine disease: response and toxicity of small molecule tyrosine kinase inhibitors in patients with thyroid carcinoma: a systematic review and meta-analysis. Eur J Endocrinol. 2015;172(5):R215-R225.

61. Smith DC, Smith MR, Sweeney C, et al. Cabozantinib in patients with advanced prostate cancer: results of a phase II randomized discontinuation trial. J Clin Oncol. 2013;31:412-419.

62. Leibowitz-Amit R, Pintilie M, Khoja L, et al. Changes in plasma biomarkers following treatment with cabozantinib in metastatic castrationresistant prostate cancer: a post hoc analysis of an extension cohort of a phase II trial. J Transl Med. 2016;14:12.

63. Lee RJ, Saylor PJ, Michaelson MD, et al. A dose-ranging study of cabozantinib in men with castration-resistant prostate cancer and bone metastases. Clin Cancer Res. 2013;19(11):3088-3094.

64. ClinicalTrials.gov [webpage on the Internet]. Cabozantinib-s-malate or Sunitinib Malate in treating patients with previously untreated locally advanced or metastatic kidney cancer. 2016. Available from: https:// clinicaltrials.gov/ct2/show/NCT01835158. Accessed May 29, 2016.

65. Nguyen L, Benrimoh N, Xie Y, Offman E, Lacy S. Pharmacokinetics of cabozantinib tablet and capsule formulations in healthy adults. Anticancer Drugs. 2016;27(7):669-678.

66. Belum VR, Serna-Tamayo C, Wu S, Lacouture ME. Incidence and risk of hand-foot skin reaction with cabozantinib, a novel multikinase inhibitor: a meta-analysis. Clin Exp Dermatol. 2016;41(1):8-15.

67. Najjar YG, Mittal K, Elson P, et al. A 2 weeks on and 1 week off schedule of sunitinib is associated with decreased toxicity in metastatic renal cell carcinoma. Eur J Cancer. 2014;50(6):1084-1089.

68. Bracarda S, Iacovelli R, Boni L, et al; Rainbow Group. Sunitinib administered on 2/1 schedule in patients with metastatic renal cell carcinoma: the RAINBOW analysis. Ann Oncol. 2015;26(10):2107-2113.

69. Kalra S, Rini BI, Jonasch E. Alternate sunitinib schedules in patients with metastatic renal cell carcinoma. Ann Oncol. 2015;26(7): 1300-1304.

70. Kondo T, Takagi T, Kobayashi H, et al. Superior tolerability of altered dosing schedule of sunitinib with 2-weeks-on and 1-week-off in patients with metastatic renal cell carcinoma-comparison to standard dosing schedule of 4-weeks-on and 2-weeks-off. Jpn J Clin Oncol. 2014; 44(3):270-277.

71. Guida FM, Santoni M, Conti A, et al. Alternative dosing schedules for sunitinib as a treatment of patients with metastatic renal cell carcinoma. Crit Rev Oncol Hematol. 2014;92(3):208-217.

72. ClinicalTrials.gov [webpage on the Internet]. Cabozantinib-s-malate and nivolumab with or without ipilimumab in treating patients with metastatic genitourinary tumors. 2016. Available from: https://clinicaltrials. gov/ct2/show/NCT02496208. Accessed May 29, 2016.

73. Motzer RJ, Escudier B, McDermott DF, et al. CheckMate 025 Investigators. Nivolumab versus everolimus in advanced renal-cell carcinoma. N Engl J Med. 2015;373(19):1803-1813.

74. Apolo AB, Tomita Y, Lee M-J, et al. Effect of cabozantinib on immunosuppressive subsets in metastatic urothelial carcinoma. ASCO Annual Meeting. 2014. J Clin Oncol. 32:5s, 2014 (suppl; abstr 4501). 
75. Motzer RJ, Hutson TE, Glen H, et al. Lenvatinib, everolimus, and the combination in patients with metastatic renal cell carcinoma: a randomised, phase 2, open-label, multicentre trial. Lancet Oncol. 2015;16:1473.

76. ClinicalTrials.gov [webpage on the Internet]. Cabozantinib-S-malate, crizotinib, volitinib, or sunitinib malate in treating patients with locally advanced or metastatic kidney cancer. 2016. Available from: https:// clinicaltrials.gov/ct2/show/NCT02761057. Accessed May 29, 2016.

77. Haas NB, Manola J, Uzzo RG, et al. Initial results from ASSURE (E2805): adjuvant sorafenib or sunitinib for unfavorable renal carcinoma, an ECOG-ACRIN-led, NCTN phase III trial. Proceedings of the 2015 Genitourinary Cancers Symposium. Presented February 26, 2015. Chicago, Illinois: 2015. J Clin Oncol. 33, 2015 (suppl 7; abstr 403).

78. ClinicalTrials.gov [webpage on the Internet]. Sorafenib in treating patients at risk of relapse after undergoing surgery to remove kidney. 2016. Available from: https://clinicaltrials.gov/ct2/show/ NCT00492258. Accessed May 29, 2016.

79. ClinicalTrials.gov [webpage on the Internet]. Adjuvant axitinib therapy of renal cell cancer in high risk patients (ATLAS). 2016. Available from: https://clinicaltrials.gov/ct2/show/NCT01599754. Accessed May 29, 2016.

80. ClinicalTrials.gov [webpage on the Internet]. A clinical trial comparing efficacy and safety of sunitinib versus placebo for thetreatment of patients at high risk of recurrent renal cell cancer (S-TRAC). 2016 Available from: https://clinicaltrials.gov/ct2/show/NCT00375674. Accessed May 29, 2016.
81. ClinicalTrials.gov [webpage on the Internet]. Cabozantinib in patients with RET fusion-positive advanced non-small cell lung cancer and those with other genotypes: ROS1 or NTRK fusions. 2016. Available from: https://clinicaltrials.gov/ct2/show/NCT01639508. Accessed May 29, 2016.

82. Choueiri TK, Vaishampayan U, Rosenberg JE, et al. Phase II and biomarker study of the dual MET/VEGFR2 inhibitor foretinib in patients with papillary renal cell carcinoma. J Clin Oncol. 2013;31(2): $181-186$.

83. Yin X, Zhang T, Su X, et al. Relationships between chromosome 7 gain, MET gene copy number increase and MET protein overexpression in Chinese papillary renal cell carcinoma patients. PLoS One. 2015;10(12):e0143468.

84. Apollo AB, Tomita Y, Lee M-J, et al. Effect of cabozantinib on immunosuppressive subsets in metastatic urothelial carcinoma [abstract]. Proceedings of 2014 American Society of Clinical Oncology, 2014, May 30th to June 3rd. Chicago, Illinois: ASCO; 2014:Abstract nr4501.

85. Exelixis, Inc. 2016 [homepage on the internet]. Available from: http:/ www.exelixis.com/. Accessed September 20, 2016.
OncoTargets and Therapy

\section{Publish your work in this journal}

OncoTargets and Therapy is an international, peer-reviewed, open access journal focusing on the pathological basis of all cancers, potential targets for therapy and treatment protocols employed to improve the management of cancer patients. The journal also focuses on the impact of management programs and new therapeutic agents and protocols on

\section{Dovepress}

patient perspectives such as quality of life, adherence and satisfaction. The manuscript management system is completely online and includes a very quick and fair peer-review system, which is all easy to use. Visit http://www.dovepress.com/testimonials.php to read real quotes from published authors. 